\title{
Hip disorders in cerebral palsy patients: diagnostic and therapeutic approaches
}

\author{
Bülent Karslığlu, Ali Çağrı Tekin, Ersin Taşatan, Fatih Mehmet Erkin
}

Department of Orthopaedics and Traumatology, Okmeydanı Training and Research Hospital, Istanbul, Turkey

DOI: $10.18621 /$ eurj.350577

\begin{abstract}
Cerebral palsy $(\mathrm{CP})$ is a neurological disorder of central nervous system that can result with devastating outcomes. Besides all other clinical findings, hip disorders also are common in children with CP. Clinical hip manifestations include wide spectrum from hip subluxation or dislocation to dislocation with painful degeneration. If untreated they may progress to more serious level like painful hip, severe hip contractures or windswept deformity and cause hygiene problems. We aimed to present current and widely accepted information about diagnostic and therapeutic approaches of hip disordes in the patients with CP.
\end{abstract}

Keywords: Cerebral palsy, hip, disorder, children, surgery

Received: November 10, 2017; Accepted: March 21, 2018; Published Online: March 26, 2018

$\mathrm{C}$ erebral palsy (CP) is a neurological disorder of central nervous system that can result with devastating outcomes. This situation is a static, nonprogressive encephalopathy but associated musculoskeletal pathology is usually progressive. Besides all other clinical findings, hip disorders also are common in children with CP. Hip disorders are second most common clinical manifestation after equinus foot deformity [1]. Hip displacement incidence varies from $1 \%$ in the patients with spastic hemiplegia to $75 \%$ in the patients with spastic quadriplegia [2].

Clinical hip manifestations include wide spectrum from hip subluxation or dislocation to dislocation with painful degeneration [3]. Pathomechanism of spastic hip dysplasia in CP differs from developmental dysplasia of the hip (DDH). Unlike DDH, children with CP have normal hip at birth and up to age of 18 months, hip problems occur due to muscle imbalance and are progressive. If untreated they may progress to more serious level like painful hip, severe hip contractures or windswept deformity and cause hygiene problems.

At this review, we aimed to present current and widely accepted information about diagnostic and therapeutic approaches of hip disordes in the patients with CP.

Epidemiology, Pathophysiology and Natural History

$\mathrm{CP}$ is an important cause of childhood physical disability and its prevalence ranges from 1.5 to 2.5 children per 1000 live births in developed countries [4]. Most common deformity seen in children with CP is equinus deformity. Hip disorders are second most seen clinical disorders. Children have significant hip subluxation by the age of 5 years. Disability to rise to standing position by 3 years age correlates with hip subluxation [5]. Progressive hip dislocation or subluxation risk for children with $\mathrm{CP}$ is $3-47 \%$.

Address for correspondence: Bülent Karslıŏlu, MD., Okmeydanı Training and Research Hospital, Department of Orthopaedics and Traumatology, Kaptan Paşa Mahallesi, Darülaceze Cad. No:27, 34384 Okmeydant-Şişli, İstanbul, Turkey

E-mail: bukars@gmail.com 
Subluxation or dislocation occurs often posteriorly. Anterior dislocations are small portions as $1.5 \%$ of patients [3].

Classification systems are necessary for the communication between physicians, to assess development or improvement with time or treatment. The Gross Motor Function Classification System (GMFCS) is common classification system used for assessing child's movement ability based on their selfinitiated movement with emphasis on sitting, walking, and wheeled mobility. System consists of 5 levels ranging from Level 1, children with minimal or no disability and Level 5 , children with totally dependent an external assistance. Within each of the five levels, descriptions of motor function are given for several age bands in the following ranges: before age 2, 2 to 4 years, 4 to 6 years, 6 to 12 years, and 12 to 18 years (12 to 18 years assessments in GMFCS Expanded and Revised) [6].

Painless and stable hip is needed for walking as well as sitting. Severity of hip spasticity determines the prevalence of hip subluxation or dislocation. Spastic quadriplegic children who are unable to walk have highest prevalence of hip dislocation [7]. Spastic hemiplegic children, who are largest proportion of $\mathrm{CP}$ patients, usually have few hip problems but more problems at the distal part of lower extremity. Spastic diplegic patients have usually hip problems at later ages . They can walk but gait quality may be affected by hip problems [8].

Primary cause for dislocation or subluxation is spasticity and muscle imbalance. Hip problems occur because of vicious circle. Spasticity causes contractures, contractures cause dislocation or subluxation and this situation aggravates spasticity. At least bony deformities occur and clinical cycle is so completed.

\section{Diagnosis}

\section{Physical Examination}

Hip is stable at birth but years later dislocation occurs in $\mathrm{CP}$ patients. Although hip is unstable at $\mathrm{DDH}$, dislocation occurs at prenatal or perinatal period. Hip joint is normal at birth but spastic muscle imbalance and lack of weight bearing leads hip problems and so $\mathrm{CP}$ becomes obvious by the age of 6 -
9 months. Excessive femoral anteversion, posterolateral acetabular dysplasia, and flexionadduction contractures are responsible for hip problems [9]. Physician may observe preferential use of limb and asymmetry or gross motor developmental delay [10]. Goals of physical examination in $\mathrm{CP}$ patients should be to determine grades of muscle strength, to evaluate muscle tone and determine the clinical type, to evaluate degree of deformity or contracture of each joint and finally to evaluate balance and phase of walking.

Physical examination consists of neurological examination, orthopedic examination and functional examination. Hip examination is an important part of orthopedic examination. Hip abduction, adduction, flexion and extension should be evaluated separately in a detailed way. Adduction contracture is most common hip deformity in CP patients. This hip problem cause hip subluxation, scissoring of the legs and perineal hygiene problems. Second most common hip deformities in CP patients are flexion contractures that bring the body's center of gravity anteriorly and so results with increased lomber lordosis, knee flexion and ankle dorsiflexion [10]. Flexion contractures occur due to increased spasticity in the flexor muscle groups in the early period.

Hip flexion contracture can be determined with Thomas, Ely and Staheli tests. Thomas test is applied by flexing both hips while patient is supine position. One leg stays in flexion and the other leg is brought to extension and adduction. The angle between examination table and extended hip is measured. This angle represents hip flexion contracture.

Ely test is applied at prone position. One hip is stabilized manually and ipsilateral leg moved into flexion position by bending the knee. If the hip elevates from examination table that means quadriceps muscle is spastic or strained.

While applying Staheli test children's body and pelvis stays on the examination table meanwhile legs hang down from the table at prone position. If hips can't be brought to fully extended position one by one, angle between thigh and ground represents amount of flexion contracture.

Leg-length discrepancy (LLD) because of hip disorder is a common problem in $\mathrm{CP}$ patients. Coexistence of LLD and impaired motor control cause 
gait pattern disturbance. Special hip disorder patterns can cause LLD. Windswept hip is a situation at which one hip at adduction deformity and the other hip at abduction deformity. This special case comes out because of asymmetric activity of the adductors, abductors, internal and external rotator muscle. The child with windswept hip has pseudo-Galeazzi sign because of adduction contracture that causes LLD [11].

\section{Radiologic Evaluation}

Early detection of hip displacement is important for early treatment but diagnose with only clinical examination is so difficult. There is a hypotonic phase before pathologic forces begun [3]. Physicians must not miss out this period. For that reason, it is recommended that 6 monthly clinical examinations should be combined with radiological screenings until hip migration is stable between ages 2 and 6 years when hip subluxation typically begins. Faraj et al. [12] showed that if hip subluxates, there is a risk of a $10 \%$ increase in the migration percentage per year because of increasing adductor muscle contracture. AP Pelvis (serial), false profile view (Faux Profile) and abduction views are sufficient for evaluating hip disorders [13].

Choice of radiological measurement depends on pathology of the disease and age of patient. Migration percentage or migration index, Wibergs's lateral center-edge (LCE) angle and Lequesne's acetabular index are most commonly used measurement parameters in children with $\mathrm{CP}$ prior to fusion of the triradiate cartilage.

Migration percentage is the percentage of the femoral head that lies outside of the lateral borders of acetabulum. In 1980 Reimer advocated that this measurement entitled as Reimer's index, is more reliable than the LCE angle to document the extent of lateral hip subluxation [12]. First, Hilgenreiner and Perkins lines are drawn. Femoral head outside the Perkins line called as "a" and total width of femoral head called as " $b$ ". A divided by b and multiplied by 100. Migration percentage is commonly used in the management of hip disorders in CP patients. It's also used for monitoring the effect of treatments on the progression of hip subluxation such selective dorsal rhizotomy, hip abduction bracing and soft tissue surgeries. Rate of migration greater than $7 \%$ supposed to be a risk factor for progressive hip disorder [14]. Position of child's hip and pelvis especially pelvicrotations may affect the measurement results. Studies on healthy children showed a wide spectrum that can be considered as normal [14]. Upper limit of migration percentage considered as normal is $25 \%$ at 4 years age [3]. Also because of wide measurement spectrum between inter and intra measurer with repeated measurements, migration percentage has low reliability and repeatability. Measurement error for migration percentage is $\pm 10 \%$ [12].

Other radiologic measurements for evaluate the lateral coverage of femoral head are Wibergs's LCE and Lequesne's acetabular index in otherwise Tönnis angle. LCE angle is formed by the vertical line through the center of femoral head and the line from center of femoral head to the lateral sourcil. Acetabular index is formed by the line perpendicular to the horizontal axis of pelvis that passing through most medial of the sourcil and the line tangent to sourcil's most lateral tip. High acetabular index is the most powerful predictor for screening hip dislocation [3]. Below $20^{\circ}$ for LCE and above $12^{\circ}$ for acetabular index is pathological [15].

There are also lateral center-edge angle (Lequesne's) and acetabular angle. Lateral center-edge angle is used for measuring anterior hip dysplasia and acetabular angle is used for detecting acetabular inclination. They are not commonly used as the measurements mentioned above.

Although at present, X-ray measurements are the most reliable indicator of hip status, there are still some limitations in the usefulness of them because of patient's position depended nature and data we get is two-dimensional information about thethreedimensional body. Contralateral hip and knee should be maximal flexed to eliminate lomber lordosis to evaluate acetabulum accurately.

Additional imaging studies such as bomputed tomography (CT) and ultrasound may be used to get more information about pathologic hip. Threedimensional (3D) reconstruction $\mathrm{CT}$ gives true information about femoral head deformities and acetabular deficiencies. Hip subluxation or dislocations especially anterior dislocations, may easily be evaluated by the help of CT. Because at 
anterior dislocations observer may not detect migration percentage changes since at this cases hips don't dislocate laterally [3].

\section{Treatment}

\section{Non-surgical Treatments}

Early detection of hip disorders with clinical and radiological screening and set out early conservative treatment to prevent or to slow the progression of dysplasia, to improve walking ability and to provide stable sitting should be main objectives for treatment. Physical therapy is useful for children with CP in the terms of facilitate motor development, enhance their independence in motor skills, self-care, play and leisure activities [16]. Rehabilitation of a child with CP demands a multidisciplinary approach with a team that includes: pediatrician, a pediatric neurologist, an orthopedic surgeon, a physician, an occupational therapist, a pediatric physiotherapist, a child psychologist, and a social worker [17].

Many forms of physical therapy for CP suggested so far such as neurodevelopmental therapy, strength training, conductive education, passive stretching, hydrotherapy, hippotherapy, postural control or orthotic devices [18]. Although there is no evidence showing that physical therapy prevents hip subluxation alone, physical therapy may slow down subluxation. Adductor spasticity is considered as reason for hip subluxation. The purpose of physical therapy must be releasing the adductor spasticity.

Botulinum toxin A (BTX-A) also may be a part of non-surgical treatments. Injections of BTX-A reduce spasticity in the hip adductor muscles for 4 to 6 months and so increase hip range of movements. BTX-A reduces muscular hyperactivity by reversibly blocking acetylcholine release at neuromuscular junction. A study based on changes at dynamic component of muscle tone by using the data's of kneeknee distance and Ashworth scale showed a significant improvement at 4 and 12 weeks after BTX-A injection and so prevented hip migration [19]. Combined usage of BTX-A and abduction brace investigated in a study and results showed that combination reduce surgery requirement at patients with $\mathrm{CP}$. Although rate of displacement was lower in combination group, displacement continued at both groups [20]. Main disadvantage of BTX-A usage is its transient effect so that it can't be permanent treatment.

\section{Surgical Treatments}

Surgeries for CP patients are categorized as preventative (adductor release), reconstructive (femoral osteotomies) and salvage surgeries. First steps at treatment should be basic ones as muscle interventions. In young non-ambulatory $\mathrm{CP}$ patients with adduction contracture, intramuscular tenotomy of adductor longus and myotomy of gracilis muscle may avoid hip subluxation. This surgical decision is based on the theory of muscle imbalance around the hip joint, with weak abductors and extensors but relatively strong adductors and flexors.

If there is a fixed hip flexion contracture, psoas tenotomy or psoas lengthening must be added to surgical intervention. There is a controversy about the efficiency of complete psoas tenotomy at lesser trochanter level and lengthening in an intramuscular location at pelvic brim but tenotomy at the lesser trochanter level causes more robust improvement at hip extension in ambulatory $\mathrm{CP}$ patients according to last studies [21]. Besides if hamstring lengthening is planned for knee flexion contracture, psoas tenotomy also must be done because crouch gait pattern occurs secondary to hip flexion. This mixed surgical intervention skein leads to a new modality: multilevel surgery which means all the contractures of the lower extremity joints must be corrected simultaneously. Nowadays, multilevel orthopedic surgery in the treatment of children with CP has become standard treatment modality at most of institutes [22].

Although preoperative level of migration $(<33 \%$ is optimal), GMCSF level and patient's age (before 4 ages) are important factors that influence success rate of soft tissue surgery, radiologic hip subluxation improves in approximately $33 \%$ of patient with $\mathrm{CP}$ after soft tissue surgeries [23]. For that reason, patients may require bony surgeries such as femoral varusderotational osteotomy or pelvic osteotomies several years after soft tissue surgery.

Hip reconstruction is indicated for 4 years of age or older $\mathrm{CP}$ patients with $>60 \%$ migration index but who have not yet started degenerative changes of the femoral head. A study showed that loss of correction of the neck-head angle occurs in $96 \%$ of the children operated less than 4-years-old [24]. Also, bony interventions may be suitable for patients younger than 
8 years of age with previous failed soft tissue surgery result and older than 8 years of age with an migration index $>40 \%$ but no signs of degenerative changings at femoral head [3].

Bony procedures usually are used for to correct abnormal femoral anteversion, neck-shaft angle and the acetabular deformity. These procedures include femoral and acetabular osteotomies. Varisationderotation osteotomy (VDRO) of the femur is used to treat internal rotation gait in $\mathrm{CP}$ patients. Soft tissue lengthening is done to achieve at least 450 hip abduction otherwise VDRO surgery results with adduction contracture. Shortening the femur for easy reduction of the femoral head and derotating to gain physiological anteversion is main subject of VDRO surgery. Femoral osteotomy is made for to gain approximately $115-120^{\circ}$ neck-shaft angle and $15-20^{\circ}$ anteversion for an anatomically normal gait. Stable fixation of femoral osteotomy is an important factor for immediate mobilization and therapy.

The acetabulum should also be redesigned for an anatomical gait unless triradiate cartilage is closed. Migration percentage is key point for acetabular correction decision. Acetabular osteotomy should be performed when the migration percentage is over 50 $70 \%$ [25]. Pelvic osteotomies like Degaacetabuloplasty remodel the acetabulum and hip load center. Combined application of femoral osteotomy and acetabular osteotomy as one-stage procedure for hip dislocation-subluxation at cerebral palsy patient is preferable and their results are far better than femoral osteotomy alone [26].

Palliative procedures like resection arthroplasty (Castle procedure), valgus osteotomy of the femur, total hip replacement and hip arthrodesis are used for non-ambulatory patients when other methods are ineffective for decreasing hip pain due to articular cartilage loss, gaining stable sitting comfort and poor perineal hygiene in the presence of there are no other options. There are still controversies about the indications of these procedures. It is a hard and complicated decision because there is no way to get back.

\section{CONCLUSION}

It is hard to deal with hip disorders in cerebral palsy patients. The aim is to gain a stable, painless hip that allows perineal hygiene and comfortable sitting. There are lots of choices but the best choice is the choice the fits the best to the patients GMFCS situation.

\section{Conflict of interest}

The authors disclosed no conflict of interest during the preparation or publication of this manuscript.

\section{Financing}

The authors disclosed that they did not receive any grant during conduction or writing of this study.

\section{REFERENCES}

[1] Cornell MS. The hip in cerebral palsy. Dev Med Child Neurol 1995;37:3-18.

[2] Dobson F, Boyd RN, Parrott J, Nattrass GR, Graham HK. Hip surveillance in children with cerebral palsy. Impact on the surgical management of spastic hip disease. J Bone Joint Surg Br 2002;84:7206.

[3] Flynn JM, Miller F. Management of hip disorders in patients with cerebral palsy. J Am Acad Orthop Surg 2002;10:198-209.

[4] Portinaro N, Panou A, Gagliano N, Pelillo F. D.D.S.H.: developmental dysplasia of the spastic hip: strategies of management in cerebral palsy. A new suggestive algorithm. Hip Int 2009;19 Suppl 6:S69-74.

[5] Scrutton D, Baird G. Surveillance measures of the hips of children with bilateral cerebral palsy. Arch Dis Child 1997;76:381-4.

[6] Rosenbaum PL, Walter SD, Hanna SE, Palisano RJ, Russell DJ, Raina P, et al. Prognosis for gross motor function in cerebral palsy: creation of motor development curves. JAMA 2002;288:1357-63.

[7] Howard CB, McKibbin B, Williams LA, Mackie I. Factors affecting the incidence of hip dislocation in cerebral palsy. J Bone Joint Surg Br 1985;67:530-2.

[8] Hoffer MM. Management of the hip in cerebral palsy. J Bone Joint Surg Am 1986;68:629-31.

[9] Heimkes B, Martignoni K, Utzschneider S, Stotz S. Soft tissue release of the spastic hip by psoas-rectus transfer and adductor tenotomy for long-term functional improvement and prevention of hip dislocation. J Pediatr Orthop B 2011;20:212-21.

[10] Agarwal A, Verma I. Cerebral palsy in children: an overview. J Clin Orthop Trauma 2012;3:77-81.

[11] Nwaobi OM, Sussman MD. Electromyographic and force patterns of cerebral palsy patients with windblown hip deformity. J Pediatr Orthop 1990;10:382-8.

[12] Faraj S, Atherton WG, Stott NS. Inter- and intra-measurer error in the measurement of Reimers' hip migration percentage. J Bone Joint Surg Br 2004;86:434-7.

[13] Parrott J, Boyd RN, Dobson F, Lancaster A, Love S, Oates J, et al. Hip displacement in spastic cerebral palsy: Repeatability of radiologic measurement. J Pediatr Orthop 2002;22:660-7.

[14] Pountney T MA. Repeatability and limits of agreement in hip migration percentage in children with bilateral cerebral palsy. Physiotherapy 2003:89;276-81.

[15] Werner C RL. Normal values of Wiberg's lateral center-edge angle 
and Lequesne's acetabular index - a coxometric update. Skeletal Radiol 2012;41:1273-8.

[16] Ketelaar M, Vermeer A, Hart H, van Petegem-van Beek E, Helders PJ. Effects of a functional therapy program on motor abilities of children with cerebral palsy. Phys Ther 2001;81:1534-45.

[17] Gunel MK MA, TarsusluT, Livanelioglu A. Relationship among the Manual Ability Classification System (MACS), the Gross Motor Function Classification System (GMFCS), and the functional status (WeeFIM) in children with spastic cerebral palsy. Eur J Pediatr. 2009;168:477-85.

[18] Anttila H, Autti-Ramo I, Suoranta J, Makela M, Malmivaara A. Effectiveness of physical therapy interventions for children with cerebral palsy: a systematic review. BMC Pediatr 2008;8:14.

[19] Mall V, Heinen F, Siebel A, Bertram C, Hafkemeyer U, Wissel J, et al. Treatment of adductor spasticity with BTX-A in children with CP: a randomized, double-blind, placebo-controlled study. Dev Med Child Neurol 2006;48:10-3.

[20] Graham HK BR, Carlin JB, Dobson F, Lowe K, Nattrass G, et al. Does botulinum toxin a combined with hip bracing prevent hip displacement in children with cerebral palsy and 'hips at-risk'? A randomized controlled trial. J Bone Joint Surg Am 2008:90;22-33.
[21] Bialik GM, Pierce R, Dorociak R, Lee TS, Aiona MD, Sussman MD. Iliopsoas tenotomy at the lesser trochanter versus at the pelvic brim in ambulatory children with cerebral palsy. J Pediatr Orthop 2009;29:251-5.

[22] Koca K, Yildiz C, Yurttas Y, Balaban B, Hazneci B, Bilgiç S, et al. [Outcomes of multilevel orthopedic surgery in children with cerebral palsy]. Eklem Hastalik Cerrahisi 2011;22:69-74. [Article in Turkish]

[23] Stott NS, Piedrahita L; AACPDM. Effects of surgical adductor releases for hip subluxation in cerebral palsy: an AACPDM evidence report. Dev Med Child Neurol 2004;46:628-45.

[24] Brunner R, Baumann JU. Long-term effects of intertrochanteric varus-derotation osteotomy on femur and acetabulum in spastic cerebral palsy: an 11- to 18-year follow-up study. J Pediatr Orthop 1997;17:58591.

[25] Kim HT, Jang JH, Ahn JM, Lee JS, Kang DJ. Early results of onestage correction for hip instability in cerebral palsy. Clin Orthop Surg 2012;4:139-48.

[26] Al-Ghadir M, Masquijo JJ, Guerra LA, Willis B. Combined femoral and pelvic osteotomies versus femoral osteotomy alone in the treatment of hip dysplasia in children with cerebral palsy. J Pediatr Orthop 2009;29:779-83. 